\title{
Ribbon Fiber with Multiple Antiguided Phase-Locked Gain Cores
}

R. J. Beach, M. D. Feit, S. C. Mitchell, K. P. Cutter, J. W. Dawson, S. A. Payne, R. W. Mead, J. S. Hayden, D. Krashkevich, D. A. Alunni

This article was submitted to 2003 Photonics West, San Antonio, Texas, February 2-5, 2003

\section{November 20, 2002}




\section{DISCLAIMER}

This document was prepared as an account of work sponsored by an agency of the United States Government. Neither the United States Government nor the University of California nor any of their employees, makes any warranty, express or implied, or assumes any legal liability or responsibility for the accuracy, completeness, or usefulness of any information, apparatus, product, or process disclosed, or represents that its use would not infringe privately owned rights. Reference herein to any specific commercial product, process, or service by trade name, trademark, manufacturer, or otherwise, does not necessarily constitute or imply its endorsement, recommendation, or favoring by the United States Government or the University of California. The views and opinions of authors expressed herein do not necessarily state or reflect those of the United States Government or the University of California, and shall not be used for advertising or product endorsement purposes.

This is a preprint of a paper intended for publication in a journal or proceedings. Since changes may be made before publication, this preprint is made available with the understanding that it will not be cited or reproduced without the permission of the author.

This work was performed under the auspices of the United States Department of Energy by the University of California, Lawrence Livermore National Laboratory under contract No. W-7405-Eng-48.

This report has been reproduced directly from the best available copy.

Available electronically at http://www.doc.gov/bridge

Available for a processing fee to U.S. Department of Energy

And its contractors in paper from

U.S. Department of Energy

Office of Scientific and Technical Information

P.O. Box 62

Oak Ridge, TN 37831-0062

Telephone: (865) 576-8401

Facsimile: (865) 576-5728

E-mail: reports@adonis.osti.gov

Available for the sale to the public from

U.S. Department of Commerce

National Technical Information Service

5285 Port Royal Road

Springfield, VA 22161

Telephone: (800) 553-6847

Facsimile: (703) 605-6900

E-mail: orders@ntis.fedworld.gov

Online ordering: http://www.ntis.gov/ordering.htm

OR

Lawrence Livermore National Laboratory

Technical Information Department's Digital Library

http://www.llnl.gov/tid/Library.html 


\title{
Ribbon Fiber with Multiple Antiguided Phase-Locked Gain Cores
}

\author{
Raymond J. Beach, Michael D. Feit, Scott C. Mitchell, Kurt P. Cutter, Jay W. \\ Dawson, and Stephen A. Payne \\ Lawrence Livermoré National Laboratory, PO Box 808, Livermore, CA 94551 \\ Phone: (925) 423:8986, FAX: (925) 423-6195, email: beach2@llnl.gov
}

Richard W. Mead

Collimated Holes, Inc., 460 Division Street, Campbell, CA 95008

Joseph S. Hayden, David Krashkevich, and David A. Alunni

Schott Glass Technologies, Inc., 400 York Avenue, Duryea, PA 18642

\begin{abstract}
We report on the first experimental demonstration of a scalable fiber laser approach based on phase-locking multiple gain cores in an antiguided structure. A novel fabrication technology is used with soft glass components to construct the multiple core fiber used in our experiments. The waveguide region is rectangular in shape and comprised of a periodic sequence of gain and no-gain segments having nearly uniform refractive index. The rectangular waveguide is itself embedded in a lower refractive index cladding region. Experimental results confirm that our five-core Nd doped glass prototype structure runs predominately in two spatial antiguided modes as predicted by our modeling.
\end{abstract}

\section{Introduction}

We have defined a new power-scalable concept for fiber lasers known as the Ribbon Laser. The strategy for scaling is to include numerous cores in a single fiber, each able to deliver a certain amount of power (eventually about $100-200 \mathrm{~W}$ ). The key, of course, is to maintain a phase-locked wave front across these numerous core regions.

A simplified drawing of our Ribbon Laser, having only one transverse dimension, appears in Fig. 1 , where a transverse profile is shown. In this example, the refractive index is plotted, and it is seen to vary periodically. The two main approaches for achieving phase-locking are based on: evanescent and radiative coupling. If the effective index of the propagating mode is intermediate between the low and high indices, then the coupling is evanescent (i.e. the "tails" of the neighboring fields in each of the cores overlap). If the effective index is lower, then the field is not confined and migrates freely throughout the entire Ribbon structure.
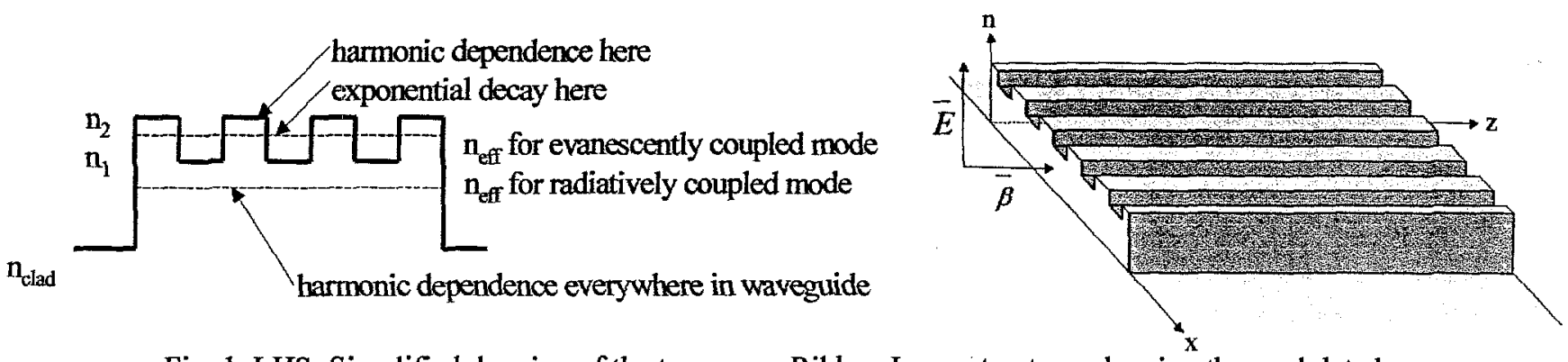

Fig. 1 LHS: Simplified drawing of the transverse Ribbon Laser structure, showing the modulated refractive index, and the $n_{\text {eff }}$ values that give rise to evanescent and radiatively coupled modes. RHS: Three-dimensional drawing of E-field, propagation direction $(+z)$, and refractive index.

We have found that a "photonic bandgap" emerges in this structure, depending on the sense of the refractive index variation. In Fig. 2 below, we see that the gain regions are the same in the upper and lower plots, although the modulation of the index has an opposite sign. On the LHS, the overlap for each of the modes is plotted against the effective index, while the fields characterizing the two modes on each 
side of the bandgap are expressly shown on the RHS. It is readily apparent that the bandgap is analogous to the valence and conduction bands of a crystal.

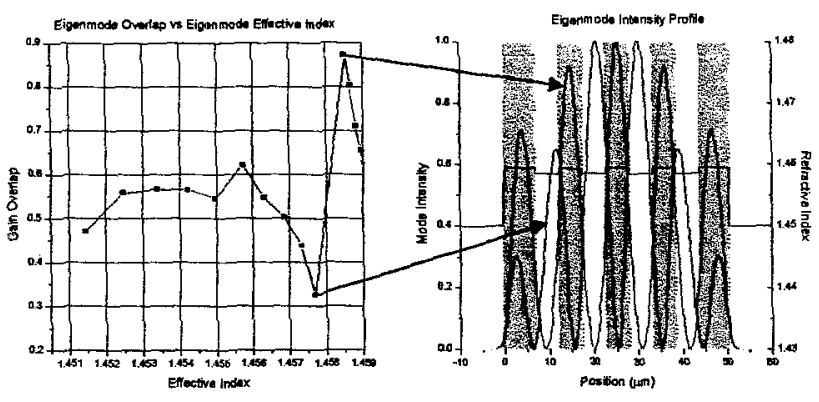

Fig. 2 Plots of the overlap of the field with the gain regions, against the effective index of the modes. The field intensities for the two modes at

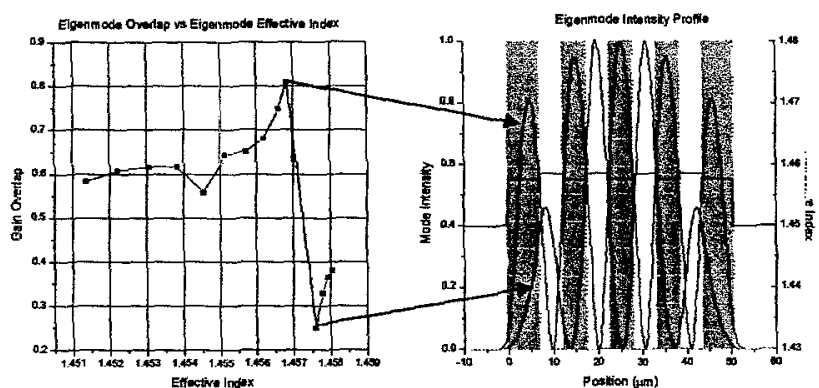
the "photonic bandgap" are plotted on the right.

The most interesting result that we obtained is pictured below in Fig. 3. Here we utilize a constant index across the Ribbon Laser, and observe that the mode structure leads to the dominance of a single mode that has considerably more overlap than all the other modes. Of course the greater overlap of this mode leads to much higher gain, such that it becomes the preferred operating mode of the laser. The preferred mode exhibits five intensity peaks, one within each of the gain regions. All of the other modes have more or less than five intensity lobes, so they have less overlap with the gain regions. This type of structure, based on having a uniform index across the ribbon structure, is analogous to a "photonic metal," since the uniform refractive index is like the constant potential seen by electrons in a metal.

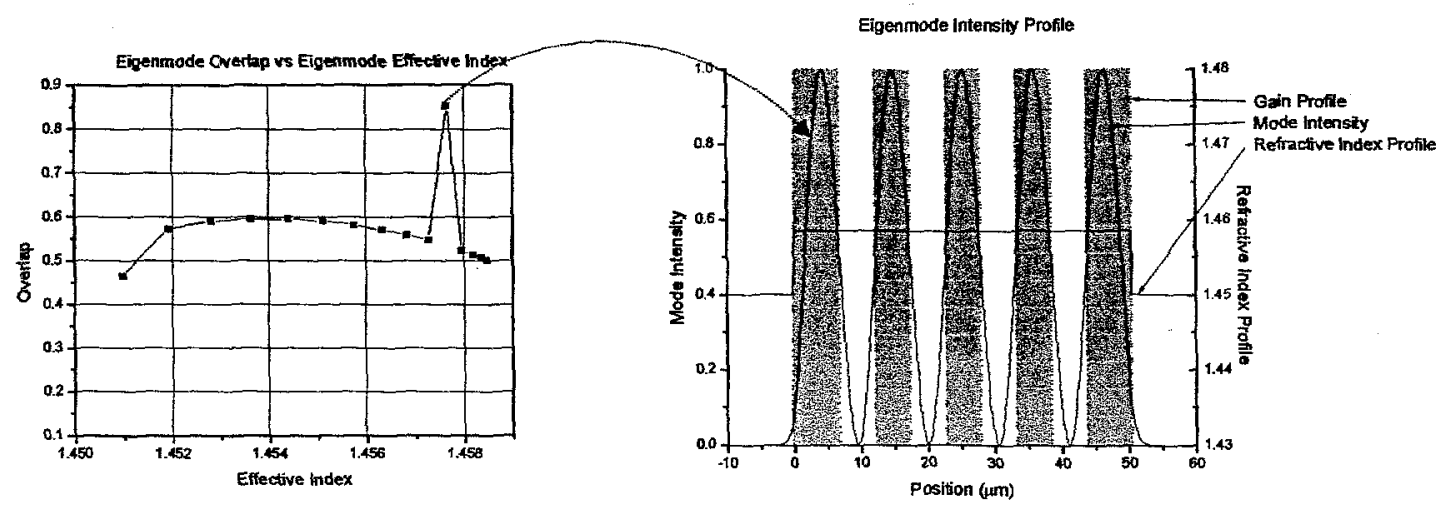

Fig. 3 Predicted modal structure for 5-core Ribbon Laser, based on a uniform transverse refractive index profile. A single preferred mode emerges that has significantly more overlap (and gain) than the other modes.

\section{Prototype Ribbon Fiber Structures}

Our proposed approach offers a pathway for scaling fiber lasers to the high average power regime based on phase locking multiple gain cores in a fiber structure using anti-guiding or radiative coupling. The initial focus is on a ribbon-like geometry surrounded by a circular pump cladding as shown in Fig. 4, in which the waveguide region contains 5 gain cores alternating with non-gain regions in a periodic array. An outer, lower index cladding surrounds the entire ribbon structure. The distinguishing feature of the Ribbon Laser 
design is a constant refractive index profile across the waveguide region as opposed to alternating higher and lower index regions. As indicated above, our modeling predicts that the constant index design will provide a mode spectrum that meets two critical design requirements: (1) strongly favored oscillation in a single transverse mode, and (2) good intensity uniformity across the waveguide structure. We require single mode operation so that a static phase corrector placed in the near field of the ribbon laser's output can optimize the phase across the aperture to achieve a high Strehl output beam. The second requirement is necessary to ensure that the gain saturates in a uniform manner across the structure so as not to increase the propensity of the device to operate in multiple transverse modes. Conceptually, the device can be scaled to higher powers simply by increasing the number of anti-guided cores within the ribbon. Modeling predicts this design will scale to 100 embedded gain cores with reasonable tolerances requirements if fused silica is used as the base material. The strong phase locking inherent in this approach should allow all the gain cores in the ribbon to communicate with each other and therefore scale to higher powers with higher phase fidelity across the aperture than is possible with similar evanescently coupled structures. This approach, in which the gain elements are radiatively coupled in a "leaky" waveguide array, is analogous to the most successful scheme for phasing laser diode elements. ${ }^{2}$

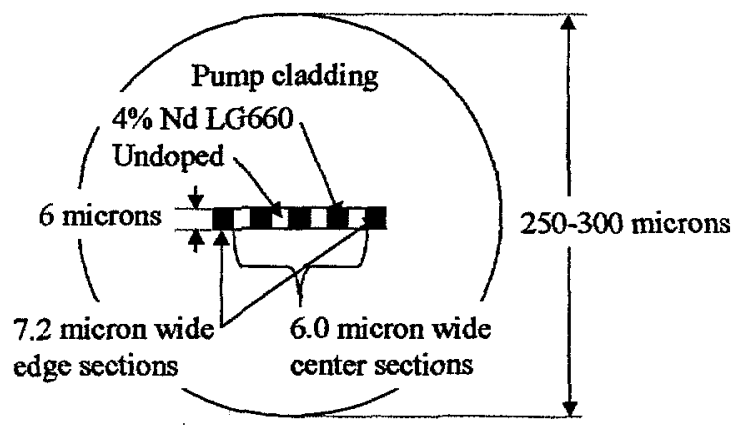

Fig. 4 Cross-sectional view of 5-core ribbon fiber being fabricated with Schott glasses. The central rectangular region is the waveguide region and the larger circular region is the pump cladding.

The structure shown in Fig. 4 uses 5 gain cores and a waveguide region that is $\sim 6 \mu \mathrm{m}$ high by $\sim 60 \mu \mathrm{m}$ wide. The pump radiation is confined to the larger $\sim 300 \mu \mathrm{m}$ diameter circular outer clad. As a material for this prototype device we have selected LG-660, an alkali-zinc-silicate laser glass, as the base glass. LG-660 offers viscosity characteristics compatible with redraw technology without risk of devitrification. A production melt of LG-660 doped with $4 \mathrm{wt} \% \mathrm{Nd}_{2} \mathrm{O}_{3}$ serves as the active core glass. Passive lanthanum containing variants of LG-660 were prepared to serve as undoped core and cladding glasses. The undoped core glass matched the active glass in refractive index and thermal expansion to $<0.001$ and $<1 \times 10^{-7} / \mathrm{K}$ respectively. On the other hand, the composition of the undoped cladding glass was adjusted to provide an index $0.006-0.007$ less than the core glasses, and with a thermal expansion roughly $2 \times 10^{-7} / \mathrm{K}$ lower than the core glasses, insuring that during fabrication of redrawn ribbon the cladding glass would be placed under compression.

Fiber Fabrication and Experimental Results

Figure 5 shows an exploded view of the preform used in the fabrication of our ribbon fiber. This structure was fabricated using bulk LG-660 undoped, and 4\% Nd-doped Silicate Schott glasses. The starting refractive index values of the various component glasses were, 1.51976 for the pump cladding, 1.52649 for the doped waveguide glass, and 1.52730 for the undoped waveguide glass. To construct the preform, square and rectangular parallelepiped waveguide component pieces were fabricated and then placed in a cut out section of the outer cylindrical cladding that was split in a clamshell configuration. The entire preform was then pieced together and held in place by inserting it in a hollow glass containment tube on which a vacuum was pulled during drawing. The preform was first drawn to cane having an outer diameter of 5 $\mathrm{mm}$ to facilitate the removal of the outside containment tube, and then the cane was drawn to fiber having a final outer diameter of 260 micrometers. No polymer coating was applied to the fiber. 


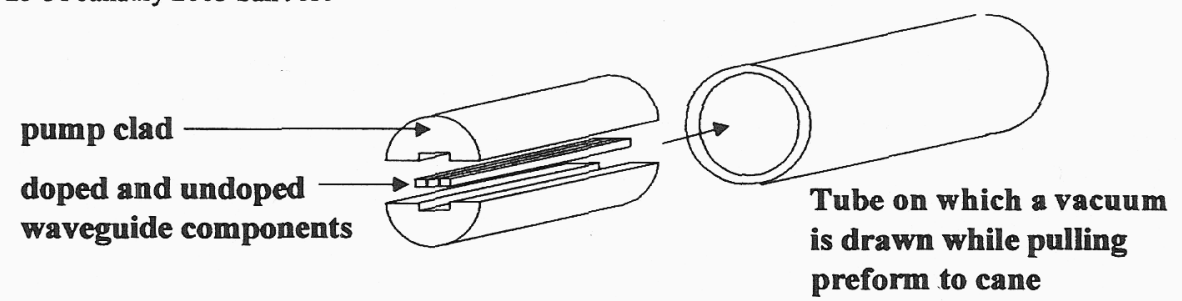

Fig. 5 Soft glasses and a "rod in tube" fabrication technology were used in the manufacture of the ribbon fiber.

The above detailed fabrication was carried out in collaboration with Schott Glass Technologies and Collimated Holes, resulting in the first prototype of the Ribbon Laser structure, shown below in Fig. 6.

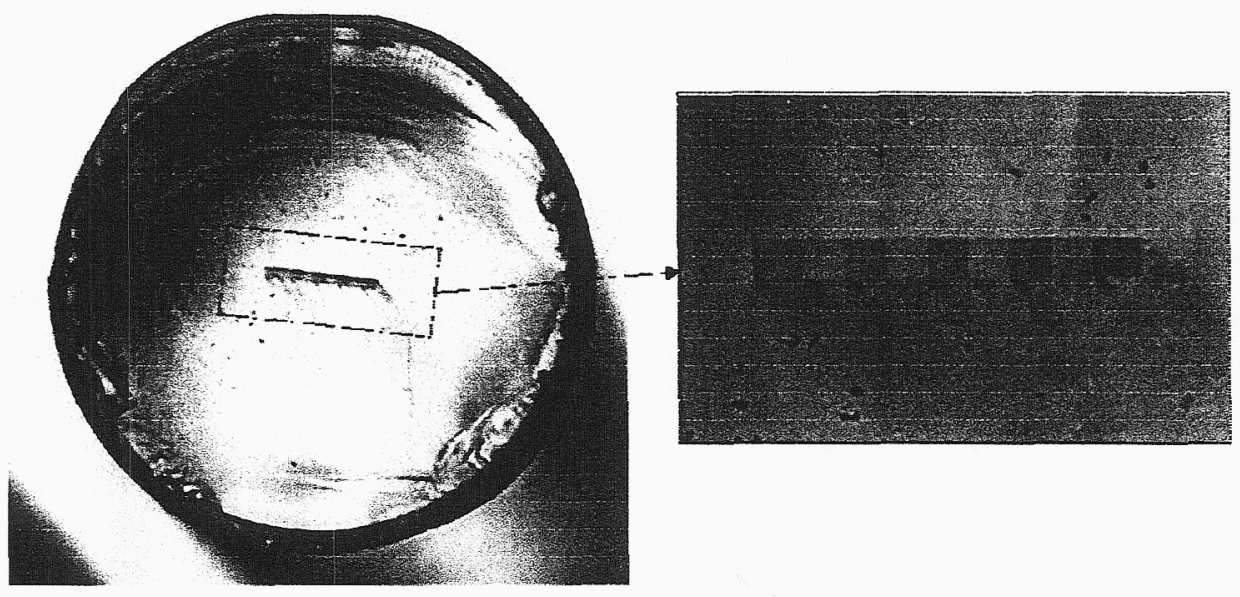

Fig. 6 Photograph of the first prototype Ribbon Laser, showing the outer pump clad, and the alternating $\mathrm{Nd}$-doped and undoped regions.

The spatial mode structure of the ribbon fiber's output beam was characterized using an end-pumping geometry in which a pulsed fiber coupled $808 \mathrm{~nm}$ diode array served as the pump source. For all measurements reported here, the laser diode pump source was pulsed on for $1 \mathrm{msec}$ at a prf of $2 \mathrm{~Hz}$. The output of the diode was imaged into the 260 micrometer diameter outer cladding of a $1 \mathrm{~m}$ long piece of the fiber having both of its ends polished normal to the fiber axis and uncoated. The pump, which singlepassed the fiber, was transmitted through a dichroic mirror contacting the fiber and having high transmission at $808 \mathrm{~nm}$ and high reflectivity at $1064 \mathrm{~nm}$. The absorption coefficient of the $\mathrm{Nd}$ doped cores is $5.6 / \mathrm{cm}$ at the $808 \mathrm{~nm}$ pump wavelength. The $1 \mathrm{~m}$ length of fiber was straight and supported by a Vgroove machined in a piece of aluminum for the characterizations reported here. Figure 7 shows a horizontal lineout of the near field intensity distribution of the fiber superimposed on the index structure of the waveguide. Although this near field intensity profile was found to be very repeatable, we did observe that arbitrarily bending or otherwise placing stress on the fiber could alter it significantly. Also shown in Fig. 7 are our model-predicted irradiance profiles calculated using the formalism presented in reference [1] and discussed below. For the index structure shown in Fig. 7, we have also calculated the entire mode spectrum supported by the waveguide as shown in Fig. 8, where mode overlap with the gain region is plotted against mode effective index value. In Fig. 8, radiative modes are defined as those with a harmonic dependence everywhere in the waveguide region, and evanescent modes as those with a harmonic dependence in some areas of the waveguide and an exponential dependence in others, depending on whether the mode index is below or above the local refractive index of the structure, respectively.

To fit our model-predicted modes to the measured near field irradiance profile we have considered the highest gain mode alone ( $5^{\text {th }}$ from the right in Fig. 8), as well as an equal intensity incoherent superposition of the two highest gain modes together $\left(5^{\text {th }}\right.$ and $6^{\text {th }}$ from the right in Fig. 8). These model predicted near field profiles are overlaid with the experimental data in Fig. 7, demonstrating that the two mode superposition replicates the prominent features of the experimental profile better than does the single mode alone. This is not surprising as the $5^{\text {th }}$ and $6^{\text {th }}$ modes are the highest gain modes supported by the structure, but are not well discriminated from each other in their gain overlap values of 0.84 and 0.80 , respectively. We believe the remaining disagreement between the experimental results and the modelcalculated near field irradiance profiles can be ascribed to a lack of detailed knowledge of the true refractive index profile of the fiber. The specific refractive index values of the various glass components used in the fiber are known to depend in detail on the cooling rate of the glass during redraw, ${ }^{3}$ as well as the 
stress induced in the structure during fabrication, both being uncharacterized for the glasses used here. While simultaneous operation in two spatial modes is evident here, we note that if a constant index throughout the waveguide region were to be obtained, then the gain discrimination between the most preferred and next most preferred mode would be greatly increased. This is shown in Fig. 8 by the dashed line and open dots, plotting mode overlap against mode effective index for the case of a constant refractive index value of 1.52649 across the entire waveguide region. In this optimized case, the gain overlap of the two highest gain modes is calculated to be 0.85 and $\mathbf{0 . 6 4}$, substantially better than the mode discrimination in our present fiber.
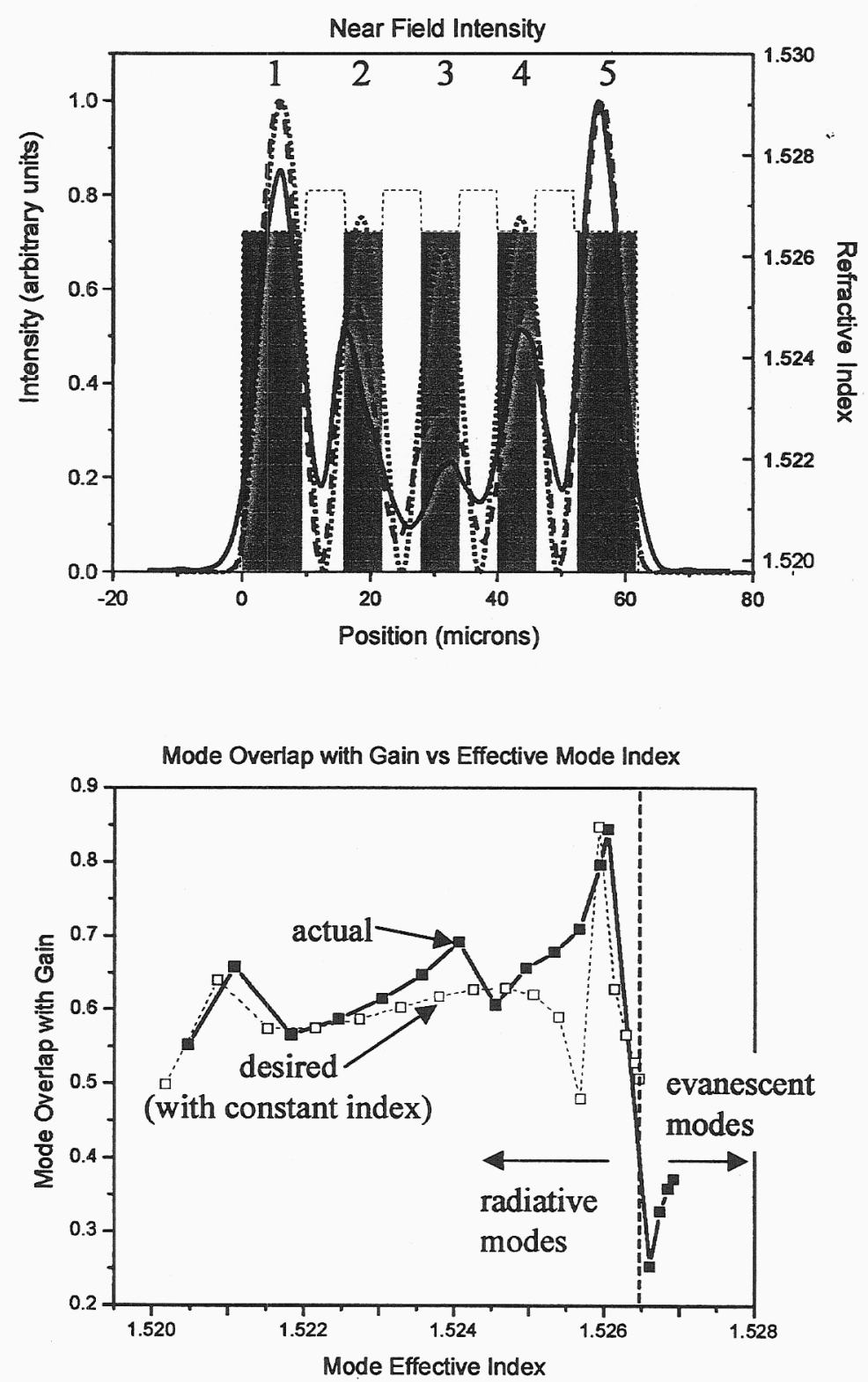

Fig. 7 Near field measured and model predicted irradiance profiles. The dotted line is our model predicted mode 5. The dashed line is our model predicted incoherent superposition of mode 5 and 6 together. The solid line is the experimentally measured near field lineout. The shaded areas denote those regions doped with $\mathrm{Nd}^{3+}$. The scale on the right references the refractive index profile, shown as a light dotted line.
Fig. 8 The dark line is a plot of mode overlap with the gain-loaded portion of the fiber against the effective index value for the various modes supported by the waveguide structure shown in Fig. 7. The dots represent the individual modes. The dashed line is the calculated mode overlap for a waveguide structure similar to the one shown in Fig. 7, but optimized with constant index across the waveguide region.

Figure 9 shows a horizontal lineout of the experimental far field irradiance profile of the fiber. Also shown in Fig. 9 is a far field profile corresponding to just the $1^{\text {st }}$ and $5^{\text {th }}$ near field lobes as defined in Fig. 7. In this case, an appropriate mask in the near field blocks all but the selected near field lobes. The far field profiles were generated by directly focusing the appropriately masked near field output of the ribbon fiber onto a camera CCD and digitizing the images, shown as inset photographs in Fig. 9. 
Farfield Intensity

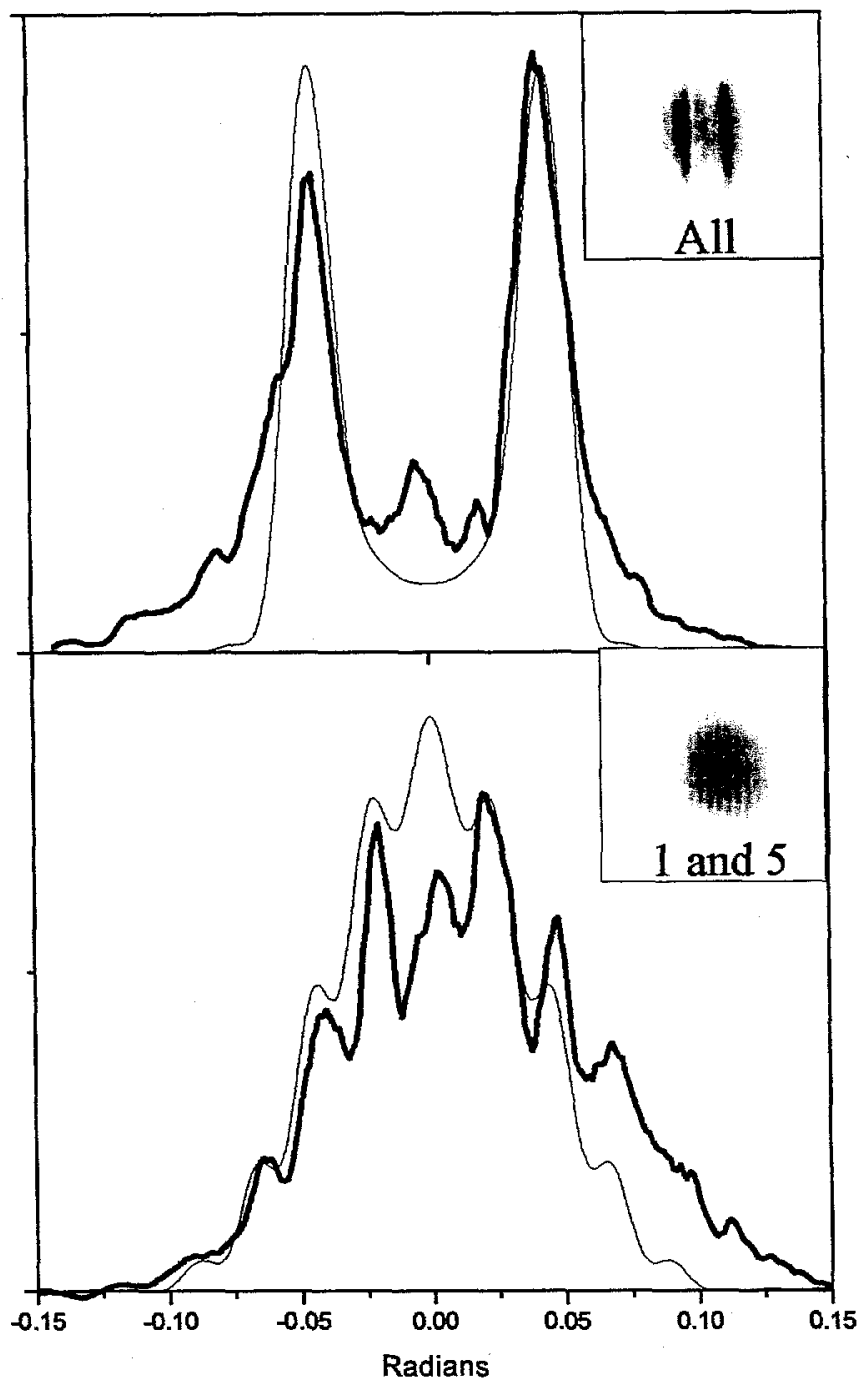

Fig. 9 The darker lines correspond to line outs of the experimentally measured far field irradiance profiles shown in the inset pictures, and the lighter lines correspond to the model calculated profiles as described in the text. The top figure is the far field from the entire aperture, and the bottom figure is the far field from near field lobes 1 and 5 only.

Also shown in Fig. 9 are the model-calculated far field profiles generated by propagating the appropriately masked, model-calculated near field. Evident in Fig. 9 is agreement between the principal features of the measured and modeled far field profiles indicating there is coherence across the lasing aperture of the ribbon fiber. Contributing to the remaining disagreement between model and experiment is our limited knowledge of the detailed refractive index values of the various waveguide components as discussed above, as well as a possible admixture of modes beyond the two used in our modeling due to mode-to-mode scattering. ${ }^{4}$ Finally, we characterized the vertical axis (narrow dimension) emission from the ribbon fiber, measuring the $\mathrm{M}^{2}$ parameter in this dimension to be 2.4 , in reasonable agreement with our model prediction of two captured modes in this dimension.

We have also used a $15 \mathrm{~cm}$ section of the ribbon fiber as the gain element in a laser oscillator to assess the energetic performance of our antiguided structure, primarily to ascertain the passive loss of the structure under lasing conditions. A $4 \%$ reflective output coupling was investigated using the uncoated end of the fiber as the output coupler, and then $10 \%, 20 \%$, and $30 \%$ reflective output couplings were 
investigated by contacting various flat mirrors to the output end of the fiber. For $4 \%$ reflectivity, the measured slope efficiency was $0.24 \mathrm{~W} / \mathrm{W}$ referenced to the absorbed pump power, and the peak opticaloptical efficiency was $0.25 \mathrm{~W} / \mathrm{W}$ at an absorbed pump power of $3.2 \mathrm{~W}$. System performance using the $10 \%, 20 \%$, and $30 \%$ reflective output couplers was almost constant with a slope efficiency of $0.32 \mathrm{~W} / \mathrm{W}$ and a peak optical-optical efficiency of $0.25 \mathrm{~W} / \mathrm{W}$. Setting the passive loss of the fiber at $0.005 / \mathrm{cm}$, we could reproduce this energetics behavior using a model based on a previously published analysis of endpumped laser systems. ${ }^{5} \mathrm{~A} 1 \mathrm{msec}$ pump pulse was used in these characterizations, and the output radiation from the fiber was observed to be unpolarized with a spectrum extending from $1059 \mathrm{~nm}$ to $1069 \mathrm{~nm}$.

$$
\rightarrow
$$

\section{Scaling to Larger Devices}

Figure 3 depicts the gain discrimination that can be realized in an ideal structure with no refractive index or size errors in the constant index rectangular waveguide region of the fiber. However, realistic scenarios must consider that geometric and index of refraction variations will have a negative impact on the gain discrimination between the different modes as the transverse size of the waveguide aperture is grown. Of the two factors, the refractive index must be the most stringently controlled in order to maintain good gain discrimination of the preferred mode over the other modes possible in the strucuture ${ }^{1}$.
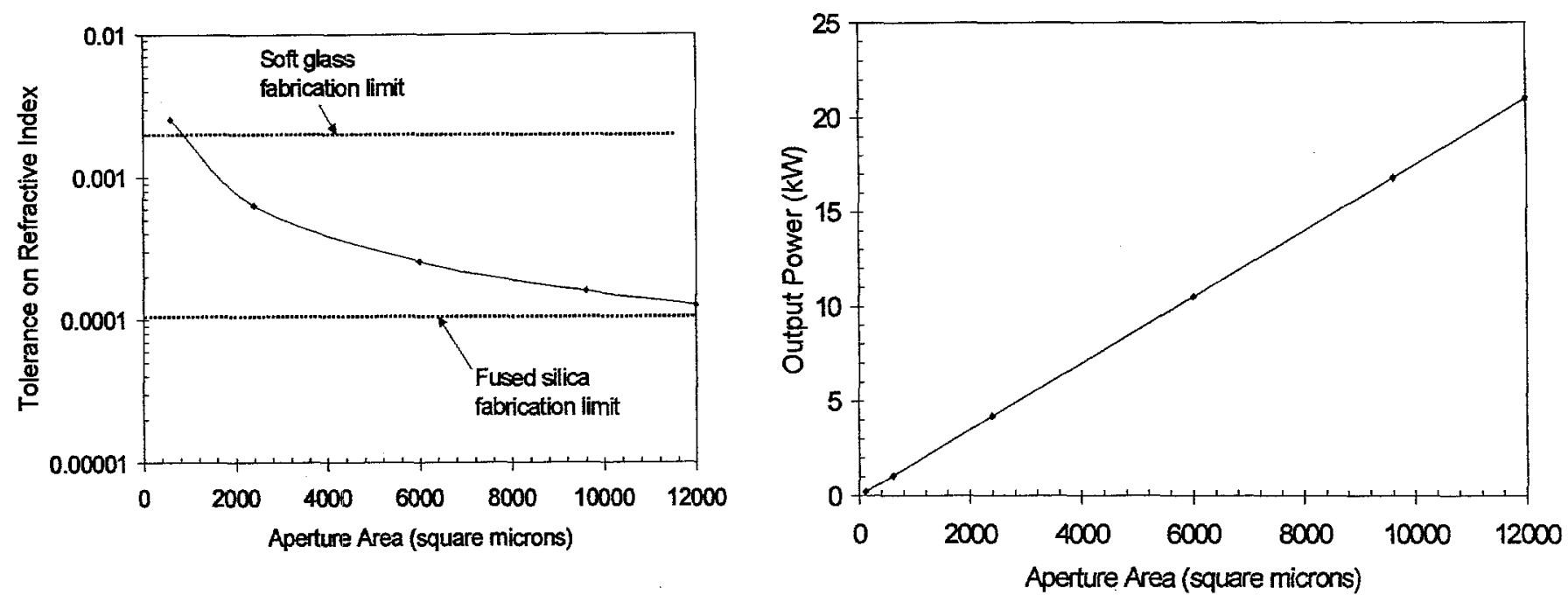

Fig. 10 Left hand side: plot of required index tolerance vs. aperture area. Right hand side: plot of output power vs. aperture area. Soft glass has a power limit of $\sim 1 \mathrm{~kW}$, while a fused silica Ribbon Laser can yield $20 \mathrm{~kW}$ based on improved refractive index control.

In Fig. 10 we have included the tolerance limits achievable with both soft glasses and fused silica, and plotted average power vs aperture size. As evident in these plots, scaling to $20 \mathrm{~kW}$ will require the use of fused silica for the fiber, and so fabrication technologies will need to be developed.

A key feature of the Ribbon Laser waveguide is the ability to decouple the inter-mode scatter from the scaling of the aperture. Inter-mode power scatter occurs between the excited mode and the modes that are most closely phase matched to the excited mode. Specifically, inter-mode power scatter is inversely proportional to the difference in effective index between two modes to the fourth power. ${ }^{4}$. This problem is seen in standard single core fibers today, when the average power is scaled through simply scaling the core size. For a circularly symmetric waveguide, the fundamental mode is the preferred mode for selective excitation. In this case, the difference in effective index between the fundamental mode and the nearest higher order mode decreases as the square root of the aperture area increases, resulting in the inter-mode power scattering increasing with the square of the aperture area. However, in the ribbon riber geometry we have another degree of freedom. By allowing the refractive index in the ribbon fiber's cladding region to vary from the top to the side of the waveguide, the ribbon fiber waveguide can be made single mode in one dimension and left multi-mode in the orthogonal dimension. This eliminates potential degeneracy between higher order modes. Figure 11 below, illustrates our proposed index profile for the ribbon fiber. 


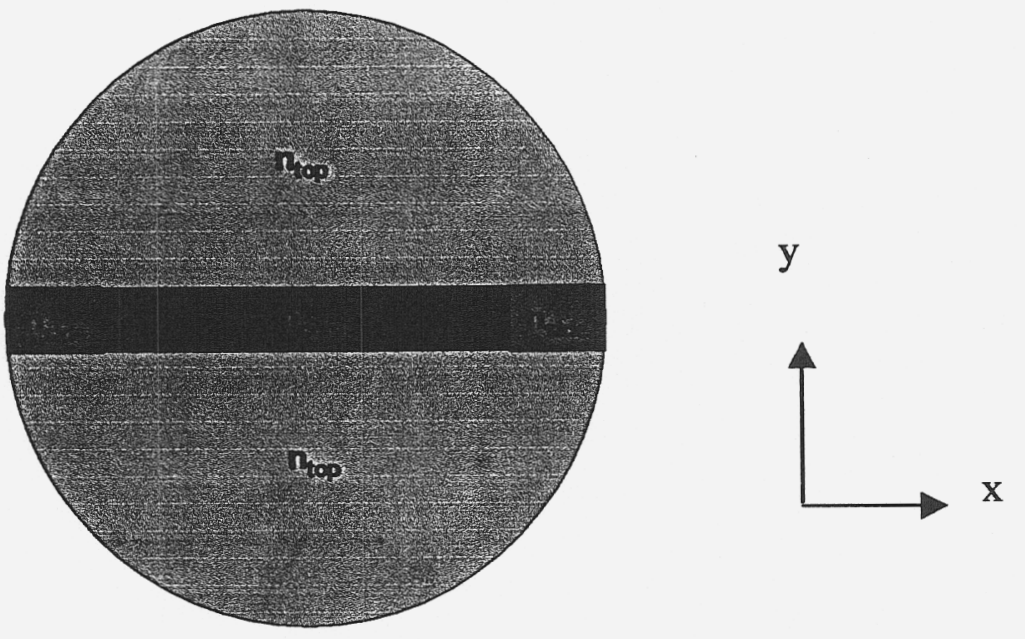

Fig. 11 A schematic example of a generic m-mode fiber refractive index profile for the Ribbon Laser.

By having a different index for the top and side claddings, we introduce an additional degree of freedom in our ability to independently engineer the mode structure in the $\mathrm{x}$ and $\mathrm{y}$-directions. Consider the modal propagation constant $\beta_{\mathrm{m}}$ of mode $\mathrm{m}$, it is related to $\mathrm{k}$, the propagation constant for light propagating in glass of index $\mathrm{n}_{\text {core }}$ and $\mathrm{k}_{\mathrm{x}}$ and $\mathrm{k}_{\mathrm{y}}$ the portions of the wavevector parallel to the $\mathrm{x}$ and $\mathrm{y}$ axes respectively by the following relation:

$$
\beta_{m}=\sqrt{k^{2}-k_{x}^{2}-k_{y}^{2}}
$$

where $\mathrm{k}=2 \pi \mathrm{n}_{\text {cord }} / \lambda$ and $\lambda$ is the free space wavelength of the beam. $\mathrm{k}_{\mathrm{x}}$ is determined for a given mode number (or number of intensity peaks) in the $\mathrm{x}$ dimension from the width of the core in the $\mathrm{x}$ dimension and $\mathrm{n}_{\text {core }}$ and $\mathrm{n}_{\text {side }} \cdot \mathrm{k}_{\mathrm{y}}$ is determined independently from $\mathrm{k}_{\mathrm{x}}$ from the height of the core in the $\mathrm{y}$ dimension and $\mathrm{n}_{\text {core }}$ and $\mathrm{n}_{\text {top }}$ and we can engineer the $\mathrm{y}$-dimension to ensure that it guides only the fundamental mode in that dimension (preferably as large a mode as possible). We are then free to adjust $n_{\text {side }}$ and $n_{\text {core }}$ to the amount required guide the preferred higher order mode for the desired aperture size. The effective index of a mode is defined as $n_{\mathrm{eff}}=\beta_{\mathrm{m}} \lambda(2 \pi)$ which is a wavelength independent way of looking at the propagation characteristics of a waveguide.

In Fig. 12 below, we plot $\mathrm{n}_{\text {eff }}$ for a waveguide with the following characteristics, width $=120 \mu \mathrm{m}$, height $=10 \mu \mathrm{m}, \mathrm{n}_{\text {core }}=1.457, \mathrm{n}_{\mathrm{top}}=1.456, \mathrm{n}_{\text {side }}=1.45$. We note that we plan to achieve the lower than silica effective indices for $\mathrm{n}_{\text {top }}$ and $\mathrm{n}_{\text {side }}$ via a photonic crystal fiber cladding around the core.

ncore=1.457, ntop=1.455, nside=1.41, width $(x$-axis $)=120$ microns, height $(y$ axis $)=10$ microns, wavelength $=1060 \mathrm{~nm}$

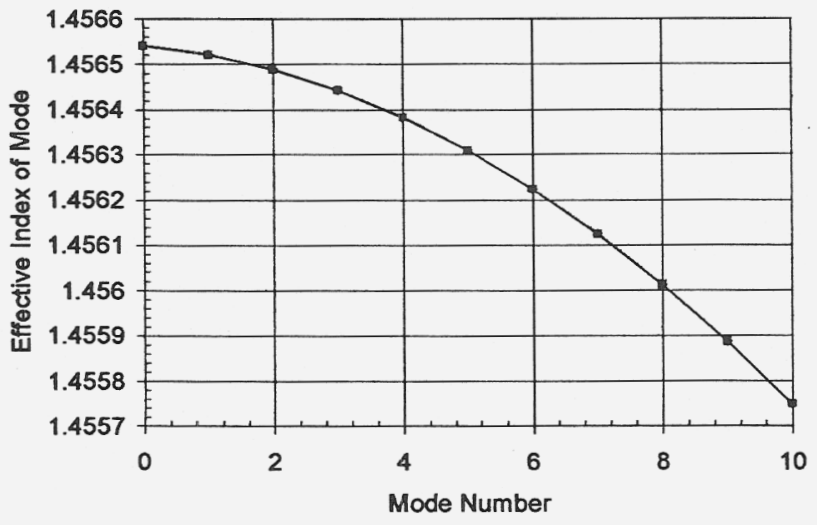

- Polarizaed Parallel to $x$-axis - Polarized Parallel to $y$-axis

NA $(x$-axis $)=0.37$

$\mathrm{NA}(\mathrm{y}$-axis $)=0.08$
Fig. 12 neff for TE and TM modes of a rectangular waveguide of the general geometry given in figure 6 with dimensions and indices noted in the text above. 
We note several features of interest from figure 12. First, the difference in $n_{\text {eff }}$ between mode $m$ and $m+1$ is increasing with increasing $\mathrm{m}$. Thus we can select the index difference between the preferred mode and its neighbor modes by choosing the appropriate mode. We plan to increase the preferred mode number faster than the aperture area so as to maintain constant index difference between the preferred mode and adjacent modes. Thus beam quality (or rather its equivalent in this case, single mode propagation length of the preferred mode) remains constant as the aperture area is scaled. This is not wholly unexpected. If we reexamine equation 1 above we see that as the mode number in the $\mathrm{x}$ dimension increases we expect $\mathrm{k}_{\mathrm{x}}$ to increase, this in turn pushes us further along the circle of radius $\left(\mathrm{k}^{2}-\mathrm{k}_{\mathrm{y}}{ }^{2}\right)^{0.5}$ that is defined by equation 1 . Near $k_{x}=0$ the slope of the circle ${ }^{3}$ s nearly flat and the change in $\beta_{\mathrm{m}}$ is small for a change in $k_{x}$, as $k_{x}$ increases the slope of the tangent to the circle increases and the same small change in $k_{x}$ generates an increasingly larger change in $\beta_{\mathrm{m}}$. Second, there is little to no birefringence or index difference between the orthogonal polarization modes. However, due to the asymmetric nature of the fiber, we can ensure that the fiber is always wound such that the $\mathrm{x}$-axis of the waveguide is perpendicular to the radius of the bend. $A$ review of the effects of bending on optical fiber birefringence ${ }^{6}$ shows that this will create birefringence within the waveguide, which can be employed for polarization maintaining operation. By selecting the winding mandrel appropriately, we can control the amount of induced birefringence such that it gives some polarization holding, but not so much that it brings another mode into effective index alignment with the preferred mode.

\section{Conclusion}

In summary, we have selected and extensively modeled a ribbon-like geometry in which the waveguide region contains multiple gain cores alternating with non-gain regions embedded in a constant index profile, yielding good mode selectivity and intensity uniformity across the waveguide structure. Additionally, we have experimentally demonstrated the use of antiguiding as a mechanism to phase lock a multiple cores in a single fiber laser. This demonstration was made using a five-core ribbon fiber structure that was fabricated using a novel soft glass fabrication technology. Although our prototype structure ran predominately in two antiguided modes rather than the desired single spatial mode because of varying refractive index across the waveguide, our experiments have demonstrated that our basic understanding of the optical physics of the device is correct. The benefit of our soft glass approach over a fused silica approach is that it greatly simplifies the fabrication of complicated fiber preforms. However, this advantage has to be weighed against the refractive index control possible with soft glasses. Based on scaling arguments presented here, which include the impact of mode to mode scattering and realistic refractive index tolerances, we believe soft glass based ribbon fibers are only scalable to the $\sim 1 \mathrm{~kW}$ average power level, while fused silica based ribbon fibers are scalable to the $20 \mathrm{~kW}$ average power level. In any case, the soft glass preform has yielded a prototype fiber that has demonstrated the essential novel device physics of a new class of structures. Due to the delocalized nature of their eigenmodes, antiguided structures similar to the ribbon fiber reported here with constant index across their waveguide region are being evaluated as potential routes to extremely high power, single spatial mode radiation.

The authors gratefully acknowledge many useful conversations with William Krupke, and Zhi Liao of LLNL, and LeAnn Brasure and Nathan Brilliant of The Air Force Research Laboratory (AFRL). This work was supported by the U.S. AFRL, Albuquerque, under Contract No. L-8958.1, and by the U.S. Department of Energy under contract W-7405-ENG-48.

\section{References}

${ }^{1}$ R.J. Beach, M.D. Feit, R.H. Page, L.D. Brasure, R. Wilcox, and S.A. Payne, "Scalable antiguided ribbon laser," JOSA B 19, 1521-1534, 2002.

${ }^{2}$ D. Botez and D. R. Scifres, Diode Laser Arrays, Chapter 1, Cambridge Univ. Press 1994.

${ }^{3}$ H.J. Hoffmann, W.W. Jochs, and N.M. Neurtoth, "Relaxation phenomena of the refractive index caused by thermal treatment of optical glasses below $T_{\mathrm{g}}$," SPIE Vol. 970, Properties and Characteristics of Optical Glass (1988). ${ }^{4}$ M.E. Fermann, "Single-mode excitation of multimode fibers with ultrashort pulses," Opt. Lett., 23, 52-54,
1998 . 
${ }^{5}$ R.J. Beach, "CW Theory of Quasi-Three Level End-Pumped Laser Oscillators," Optics Communications 123, 385-393, 1995.

${ }^{6}$ L.B. Jeunhomme, "Single-mode fiber optics: Principles and applications", Chapter 2, $2^{\text {nd }}$ edition, Marcel Dekker, Inc., 1990. 